- he conditions or syndromes to be considered in this article are summarised in table 1. Many are seen relatively infrequently in neurological practice. Among 4000 successive outpatient referrals, pupillary abnormalities or ophthalmological syndromes accounted for 39 (table 2). More often, some of these abnormalities—for example, a tonic pupil (Holmes-Adie syndrome)— are found by chance. Sometimes the abnormality has not been previously recognised or, even worse, has been misinterpreted. Documentation of such changes is important—not necessarily towards diagnosing the patient's complaint, but towards preventing misinterpretation of the problem in the future. In the acutely ill patient, the recognition of the syndromes of brain herniation, particularly uncal and trans-tentorial, are critical to patient care.

\title{
ARGYLL ROBERTSON PUPIL
}

Argyll Robertson described his pupillary syndrome in 1869. The definition should be strict otherwise a whole host of pupillary abnormalities with some degree of light-near dissociation will be so designated. The cardinal features are summarised in table 3. The pupils are not necessarily fixed to light, though they become so in the later stages. What is critical is the dissociation between the extent of the near and the light reaction. A preserved normal acuity should be included in the criteria to exclude numerous conditions in which a depression of light sensitivity-for example, caused by optic nerve disease-leads to light-near dissociation. When strictly defined in this way the condition is pathognomonic of neurosyphilis. Cases are now rare, though the recent burgeoning of cases of primary syphilis suggest that its incidence may rise in the future. The most likely site of the causative lesion is one affecting the light reflex fibres rostral to the oculomotor nucleus. ${ }^{1}$

\section{HOLMES-ADIE (TONIC) PUPIL}

Patients with a tonic pupil tend to present (if at all) because of a difference in pupillary size. The abnormality has often been pointed out to them. Sometimes the patient notices a problem in bright lights, the consequence of the failure of reflex constriction. The condition is usually unilateral and tends to predominate in young to middle aged women. ${ }^{2}$ The affected pupil reacts incompletely to both light and dark so that it is larger than its fellow in the light, but may be smaller in the dark because of the intact reflex dilatation of the unaffected pupil. The cardinal feature of the condition is the tonic near reaction. Whenever a depressed light reaction is observed in a "normal" (rather than meiotic) pupil, it is essential to observe the near reaction, and then its release, over at least a minute. The affected pupil may eventually be smaller than its fellow if the near reaction is sustained for a sufficient length of time.

Tonic pupils can show supersensitivity, with constriction, to dilute parasympathomimetic agents (for example, $0.125 \%$ pilocarpine), but it should be possible to diagnose the problem without using pharmacological agents. The accommodation component of the near reaction is also tonic.

Most cases appear spontaneously, but sometimes the condition evolves from an acute iridoplegia. The author has seen such a progression in a patient with neurosarcoidosis (fig 1).

The phenomenon is thought to be the consequence of aberrant regeneration of accommodation fibres arising from prior damage to the ciliary ganglion. In many cases the condition has not been recognised either by the patient or the referring physician. It should be carefully documented to avoid misinterpretation in the future. In some cases, the deep tendon reflexes are depressed (also

Correspondence to: Dr David Perkin, Division of Neuroscience and Psychological Medicine, Imperial College London, Charing Cross Campus, $\mathrm{St}$ Dunstan's Road, London W6 8RP, UK; d.perkin@imperial. ac.uk called the Holmes-Adie syndrome) because of interruption of synaptic transmission in the monosynaptic reflex arc in the spinal cord.

\section{HORNER'S SYNDROME}

The ocular sympathetic fibres arrive at the eye after a convoluted, though uncrossed course which begins in the hypothalamus. The characteristic features of Horner's syndrome (the consequence of interruption of these fibres) are ptosis (of mild degree), meiosis, and "upside-down" ptosis. 


\begin{tabular}{l} 
Table 1 Neuro-ophthalmological and pupillary \\
syndromes \\
\hline Argyll Robertson pupil \\
Holmes-Adie (tonic) pupil \\
Horner's syndrome \\
- Uncal herniation \\
Trans-tentorial herniation \\
Accommodation spasm \\
Superior orbital fissure syndrome \\
Cavernous sinus syndrome \\
- Mobius' syndrome \\
- Gradenigo's retraction syndrome \\
Parinaud's syndrome \\
- Kearns Sayre syndrome \\
- Eales' disease \\
\hline
\end{tabular}

Enophthalmos is not confirmed if formal measurement of the globe position is carried out. Though with some causes there is loss of sweating to the face, the finding is difficult to elicit in clinical practice. The conjunctival vessels may appear dilated for a short period soon after the onset. Though pharmacological tests have been suggested in order to distinguish pre- and postganglionic causes of the syndrome, the results are not necessarily consistent and the distinction is seldom critical in clinical practice. If necessary, the condition can be confirmed by the instillation of $4 \%$ cocaine eye drops. The normal pupil dilates, while the Horner's pupil fails to do so. At times there may be some difficulty distinguishing the meiosis of Horner's syndrome from a physiological anisocoria. If in doubt, take the patient into a dark room, preferably accompanied if the patient is of the opposite sex! The Horner's pupil fails to dilate in the dark, so that the anisocoria becomes more apparent. In the case of physiological anisocoria, both pupils dilate, so that the degree of anisocoria remains unchanged.

Horner's syndrome can be found as part of a neurological disorder (for example, the lateral medullary syndrome), but is sometimes found in isolation. ${ }^{3}$ Debate remains as to whether an isolated Horner's syndrome merits investigation. Certainly, a chest $x$ ray is reasonable under such circumstances. Although an isolated Horner's syndrome has been described as the presenting manifestation of syringomyelia, inspection of the case record indicates that the patient had also complained of episodic occipital headaches. ${ }^{4}$ My own practice is not to investigate a truly isolated Horner's syndrome other than to perform a chest $x$ ray. A painful Horner's syndrome, however, may be the sole manifestation of extracranial carotid artery dissection and warrant vascular imaging.

\section{UNCAL AND TRANS-TENTORIAL HERNIATION}

The earliest pupillary sign in uncal herniation is a dilating pupil with an impaired direct light reaction. The sign may be apparent when the patient is still only mildly drowsy. As the condition develops, the pupil becomes widely dilated and fixed. This stage can persist for some hours before being joined by other neurological signs. In the later stages of uncal herniation, ophthalmoplegia appears, accompanied by a rapid decline in the conscious level, and sometimes a hemiplegia ipsilateral to the lesion because of compression of the cerebral peduncle against the contralateral tentorial edge.
Table 2 Pupillary and ophthalmological syndromes encountered in 4000 successive outpatient referrals

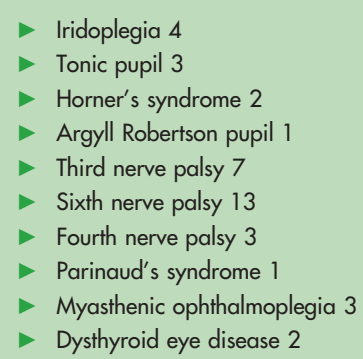

Before diagnosing an early uncal herniation syndrome, it is vital to check that pupillary asymmetry has not been recorded in the past, and that mydriatic drops have not been inserted to aid fundoscopy.

The central (transtentorial) herniation syndrome produces different signs. Though this herniation syndrome is more likely with medially placed lesions, it can equally occur with lateral masses.

The pupils remain equal, though meiosed, and, at least initially, with retained light responsiveness. The earliest eye sign tends to be impairment of upward gaze, elicited by performing the doll's head manoeuvre in the vertical plane. Later the pupils become fixed and reflex horizontal eye movements become impaired.

\section{ACCOMMODATION SPASM}

Although accommodation spasm has been described in association with organic disease (for example, myasthenia gravis) the vast majority of cases occur as part of a conversion reaction. Typically, recurring episodes of convergence, meiosis, and accommodation are encountered. During lateral gaze, the superimposition of a convergence effort produces an apparent impairment of abduction, suggesting the possibility of a sixth nerve palsy. The true cause can be identified by observing the meiosis that occurs at such times. The patient may complain of headache, poor vision or diplopia. Psychiatric referral may be necessary. Neurological investigation should be avoided.

\section{SUPERIOR ORBITAL FISSURE AND CAVERNOUS SINUS SYNDROMES}

Since these two syndromes can be difficult to distinguish clinically it is perhaps appropriate to include them under a single title-the spheno-cavernous syndrome. All the oculomotor nerves can be involved, along with the first division of the trigeminal nerve. If the lesion is in the mid to posterior portion of the cavernous sinus, the second division of the trigeminal nerve may also be affected. A Horner's syndrome can accompany these other findings. The more anterior the

Table 3 Argyll Robertson pupil
$>$ Depressed or absent light reaction
$>$ Intact near reaction
$>$ Miotic, irregular pupils
$>$ Valaterality



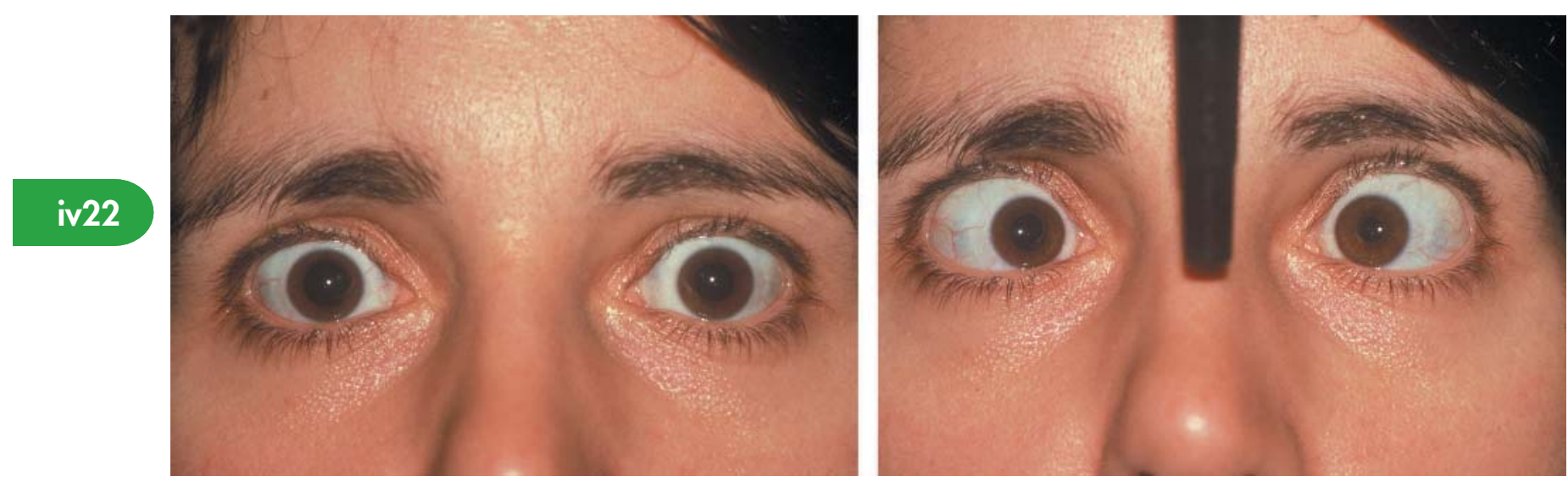

Figure 1 Sarcoidosis. Partial right iridoplegia with (left) dilated pupil and (right) incomplete near response. Reproduced with permission of the patient.

lesion, the more likely there is to be proptosis. The list of conditions that can cause this presentation is substantial. The most common candidates are, at the superior orbital fissure, a meningioma, and within the cavernous sinus, an internal carotid aneurysm. More acutely evolving causes include cavernous sinus thrombosis and carotico-cavernous fistulae.

The Tolosa-Hunt syndrome requires particular mention. ${ }^{6}$ The syndrome consists of a painful ophthalmoplegia, sometimes with oculo-sympathetic paralysis and accompanied by sensory change in the first (and sometimes the second) division of the trigeminal nerve. It is essential to realise that this presentation is not specific to a particular pathology and that the diagnosis of the Tolosa-Hunt syndrome is one of exclusion.

The condition can occur in any age group. Rarely, it may be bilateral. The pain, which is often intense and typically periorbital, tends to precede the ophthalmoplegia. Sometimes, the pathological process is more wide ranging with involvement of the optic nerve, the third division of the trigeminal, and the facial nerve. The condition tends to present acutely and is liable to recur in about half the cases. Corticosteriods dramatically alleviate the pain but it is uncertain what influence they have on the outcome of the cranial nerve palsies. Some neurological disability may persist. The pathological process appears to be a granulomatous inflammation in the region of the cavernous sinus with epithelioid cells and occasional giant cells.

Imaging usually reveals an area of abnormal soft tissue in the region of the cavernous sinus, with gadolinium enhancement on magnetic resonance imaging (MRI).

Because of the non-specific nature of the imaging changes, and because other pathological entities (for example, lymphoma) can present similarly, including an initial response to corticosteroids, protracted follow up of these cases will be necessary before the diagnosis can be finally accepted.

\section{MOBIUS' SYNDROME}

This congenital syndrome may cause consternation if it has not been previously recognised and assessed. For most patients, however, the disorder will have been recorded and misinterpretation of the findings should not occur. It is uncommon. A variety of congenital defects outside the eyes are described, including abnormalities of the face, defects of the lower cranial nerves, and limb defects. Mental retardation is usual. Typically there is a defect of horizontal gaze with preservation of vertical gaze. Occasionally there is a family history of the condition. Various underlying pathological changes have been described, typically atrophy of the relevant cranial nerve nuclei.

\section{DUANE'S RETRACTION SYNDROME}

This is most commonly the result of a congenital disorder affecting the brainstem ocular motor system. The condition is usually sporadic, but familial cases are recognised, and cases have been recognised resulting from the use of thalidomide during pregnancy. In the few cases that have come to pathological examination a variety of abnormalities have been described with typically absence of the abducens nucleus and nerve with evidence of aberrant innervation of the lateral rectus or recti by branches from the oculomotor nerves. The condition may be unilateral or bilateral, usually the former. Most patients do not report visual symptoms and vision itself is usually normal. There is limitation or absence of abduction, a variable degree of limitation of adduction, and typically a tendency to retraction of the globe with narrowing of the palpebral fissure on attempted adduction. In addition, adduction may be accompanied by some degree of vertical eye movement, typically upwards.

There may be congenital defects in other systems and in the remainder of the nervous system. The condition does not present as such. It simply needs to be recognised when encountered as a chance finding in patients presenting with other neurological problems.

\section{GRADENIGO'S SYNDROME}

As originally described, this is a syndrome triggered by mastoiditis which has extended to the petrous apex. At this site localised inflammation of the meninges is liable to affect the abducens nerve as it perforates the dura overlying the clivus. As a consequence the patient develops intense facial and ocular pain, associated with a sixth nerve palsy. There may be facial paresis in addition. Typically the pain antedates the appearance of the ophthalmoplegia. Occasionally, the meningeal infection becomes more widespread. The condition can be mimicked by other pathological processes, including aneurysm of the internal carotid artery and tumours. An alternative mechanism for abducens palsy in patients with mastoiditis is venous thrombosis of the inferior petrosal sinus. With modern imaging techniques it should be possible to distinguish these pathological entities. The syndrome is rarely encountered in neurological practice. 


\section{PARINAUD'S SYNDROME}

Parinaud's syndrome is characterised by impairment of upward gaze, accompanied by abnormalities of down gaze and convergence, eyelid retraction, and light-near dissociation. The syndrome is better termed the dorsal midbrain syndrome and is the result of lesions in the region of the posterior commissure.

Generally all forms of upward gaze are affected. The retraction of the upper lids, unassociated with any lag on down gaze, was described by Collier. Occasionally there is ptosis. Downward saccades are intact but generally slowed. In some cases, there is a paralysis of convergence, while in others, convergence spasm occurs. Convergence retractory nystagmus is an episodic retractory jerking of the eyes triggered by attempted up-gaze. It is best elicited in this situation by performing optokinetic nystagmus in the vertical plane, with the drum rotating downwards. Vertical skew is a common accompanying feature. The pupils in the dorsal midbrain syndrome are dilated and either fixed to light, or very poorly responsive to it. The near reaction is preserved.

The differential diagnosis of the syndrome is extensive. ${ }^{7}$ While classically associated with pineal tumours, virtually any pathology at the relevant site can produce the same signs. Modern imaging generally serves to make the distinction.

\section{KEARNS SAYRE SYNDROME}

Most cases of this syndrome have large mitochondrial DNA deletions. It is a progressive disorder which characteristically involves multiple systems. It is seldom encountered in neurological practice but could, theoretically at least, cause diagnostic confusion with other causes of an external ophthalmoplegia. The cardinal features are a progressive external ophthalmoplegia, pigmentary retinal degeneration and dementia, coupled with cardiac conduction defects and other systems involvement (see Overell and Lindahl, p iv53).

Ptosis usually antedates any defect of eye movement and is unilateral, but sometimes asymmetrical. It is progressive. The ophthalmoplegia is typically symmetric and consequently the patient rather seldom complains of diplopia. Eventually the ophthalmoplegia may be complete. Biopsy of skeletal muscle shows the presence of ragged red fibres and abnormal mitochondria on electron microscopy.

The pattern of retinal pigmentation differs from that seen in retinitis pigmentosa with particular involvement of the posterior fundus. Visual disability is relatively mild. Electrophysiological findings also differ from those seen in retinitis pigmentosa. Cardiac involvement takes the form of a variety of cardiac conduction defects often culminating in heart block.

Abnormal neurological findings include cerebellar ataxia and deafness. Dementia is relatively common. MRI reveals areas of white matter signal change which can be shown to correspond to spongiform degeneration at postmortem examination. There is no effective treatment.

\section{EALES' DISEASE}

Eales' disease is seldom encountered in neurological practice. Typically episodes of retinal vasculitis are accompanied by recurring retinal and vitreous haemorrhages. The disease predominates in young males and might, at least theoretically, be confused with optic neuritis. The symptoms tend to be unilateral and can include blurring of vision with decreased visual acuity. Pain on ocular movement is not a feature. The retinal findings are quite distinct from those seen in optic neuritis though it needs to be remembered that retinal venous sheathing is an accepted finding in optic neuritis, as is, on occasions, some evidence of ocular inflammatory disease. In Eales' disease, however, the changes are usually florid and not confined to the veins. The perivascular sheathing is often accompanied by flameshaped retinal haemorrhages. Peripheral hypoperfusion of the retina is the norm, but the macula is generally spared, so that central field defects are not a particular feature.

Confusion with multiple sclerosis may be furthered in those cases showing evidence of more widespread neurological involvement. That association perhaps needs to be examined critically on the basis of some of the published studies. ${ }^{8}$ In some of these cases, the retinal vessel sheathing seems to have been confined to the venous system, extensive follow up of the neurological syndrome has not been performed, and cerebrospinal fluid (CSF) studies at the time of neurological involvement are incomplete. Any neurological involvement that does occur in Eale's disease has a predilection for the dorsal cord and tends to show an incomplete recovery.

\section{SUSAC'S SYNDROME}

Susac's syndrome (microangiopathy of the brain and retina) was first recognised as a distinct entity in 1979. The condition predominates in young adult women. Retinal involvement consists of multiple branch retinal arterial occlusions. It has been suggested that cochlear involvement resulting in deafness is so common that it should be considered part of the syndrome $^{9}$ (see Overell and Lindahl, p iv53).

Neurological involvement includes behavioural disorders, pyramidal and cerebellar signs, dementia, and disturbance of sphincter function. Changes in the CSF are non-specific and there is no consistent evidence of arteritis in terms of vascular changes on formal angiography. MRI shows high signal changes on $\mathrm{T} 2$ weighted images. In the earliest report of the syndrome, the condition was attributed to cerebral lupus, but neither in the two original cases nor in subsequent cases has there been evidence to support the diagnosis of a specific collagen vascular disease. There is no firm evidence that immune modulatory therapy affects the course of the disease.

\section{REFERENCES}

1 Loewenfeld IE. The Argyll Robertson pupil, 1869-1969: a critical survey of the literature. Surv Ophthalmol 1969;14:199-299.

- A detailed analysis of the Argyll Robertson pupil and its pathogenesis.

2 Thompson HS. Adie's syndrome. Some new observations. Trans Am Ophthalmol Soc 1977;75:587-626.

3 Thompson HS. Diagnosing Horner's syndrome. Trans Am Acad Ophthalmol Otolaryngol 1977;83:840-2.

4 Kerrison JB, Biousse V, Newman NJ. Isolated Horner's syndrome and syringomyelia. J Neurol Neurosurg Psychiatry 2000;69:131-2.

A case report of an isolated Horner's syndrome secondary to syringomyelia.

5 Plum F, Posner JB. The diagnosis of stupor and coma, 3 ed. Philadelphia: FA Davis, 1980.

- A superb monograph which should be on every trainee's bookshelf.

6 Kline LB, Hoyt WF. The Tolosa-Hunt syndrome. J Neurol Neurosurg Psychiatry $2001 ; 71: 577-82$

- An extensive, recent review, though without any fresh insights as to pathogenesis.

7 Keane JR. The pretectal syndrome: 206 patients. Neurology 1990;40:684-90.

- An extensive survey of the differential diagnosis of the pretectal syndrome.

8 Singhal BS, Dastur DK. Eales' disease with neurological involvement. Part 1. Clinical features in 9 patients. J Neurol Sci 1976;27:313-21.

- The apparent association of Eales' disease with neurological pathology which suffers from the absence of modern imaging and immunological analysis.

9 Bogousslavsky J, Gaio JM, Caplan LR, et al. Encephalopathy, deafness and blindness in young women: a distinct retinocochleocerebral arteriolopathy. J Neurol Neurosurg Psychiatry 1989;52:43-6. 